\title{
Tratamiento nutricional de los síndromes colestáticos en la infancia
}

\author{
Piotr Socha \\ Departamento de Gastroenterología, Hepatología e Inmunología, Instituto Conmemorativo de Salud Infantil, \\ Varsovia, Polonia
}

\author{
Palabras clave \\ Hepatopatía $\cdot$ Vitaminas liposolubles • Vitamina E $\cdot$ \\ Desnutrición · Nutrición enteral · Nutrición parenteral • \\ Insuficiencia hepática
}

\section{Resumen}

La hepatopatía colestática causa un riesgo serio de desnutrición, que incluye la desnutrición de proteína y energía y las carencias nutricionales específicas. El estado nutricional puede evaluarse basándose en mediciones antropométricas, que pueden ser equívocas a causa de la ascitis y el edema periférico. Las determinaciones bioquímicas del estado de las vitaminas liposolubles son importantes para evaluar las necesidades de los niños. Basándose en la evaluación del estado nutricional, debe planificarse la terapia nutricional con arreglo a una pauta bien establecida. El principio básico del tratamiento nutricional consiste en corregir el estado nutricional, así como reducir el riesgo de carencias nutricionales. Los niños con colestasis precisan habitualmente un suministro extra de energía que puede obtenerse incrementando la densidad energética de los alimentos o añadiendo lípidos y polímeros de glucosa. Para recuperar el crecimiento, suele tener que incrementarse la ingestión de proteínas. El aporte complementario de vitaminas liposolubles merece atención especial y no es fácil corregir un estado carencial de vitamina $\mathrm{E}$. En algunos niños es necesario administrar la vitamina $\mathrm{K}$ por vía parenteral. Desde hace poco tiempo se utiliza con buen efecto terapéutico una vitamina
E hidrosoluble ( $d$ - $\alpha$-tocoferil polietilenglicol 1.000 succinato), administrada por vía oral. Dado que la hepatopatía progresa hacia una insuficiencia hepática en numerosas enfermedades colestáticas crónicas, el tratamiento nutricional puede considerarse a menudo como una medida para 'salir del paso' a la espera del trasplante hepático para mejorar el pronóstico. En consecuencia, el apoyo nutricional invasivo está justificado en la hepatopatía grave y comprende habitualmente la alimentación nocturna por sonda nasogástrica o incluso la nutrición parenteral.

Copyright $\odot 2009$ Nestec Ltd., Vevey/S. Karger AG, Basel

\section{Introducción}

Los requisitos de nutrientes específicos para lactantes y niños sanos son ampliamente discutidos y las recomendaciones se actualizan cada pocos años. Existen todavía muchas contradicciones en relación con algunos nutrientes. Recientemente, el aporte complementario de vitamina K en la lactancia ha sido objeto de debate. Ijland y cols. [1] pusieron en entredicho la seguridad del aporte complementario de vitamina $\mathrm{K}$ oral a una dosis diaria de 25 $\mu \mathrm{g}$ en una población sana, dada la aparición de hemorragias en 6 lactantes alimentados con leche materna en la población estudiada. Por lo visto, 5 de los 6 lactantes con hemorragia eran colestáticos y presentaban atresia biliar. Por lo tanto, la colestasis era la causa del riesgo de carencia de vitamina $\mathrm{K}$ y los resultados no necesariamente

\section{KARGER}

Fax +41613061234

E-Mail karger@karger.ch

www.karger.com
(C) 2009 Nestec Ltd., Vevey/S. Karger AG, Basel 0252-8185/08/0663-0137\$24.50/0

Accesible online en:

www.karger.com/ans
Prof. Piotr Socha

Department of Gastroenterology, Hepatology and Immunology

The Children's Memorial Health Institute, Al. Dzieci Polskich 20

PL-04-730 Warsaw (Poland)

E-Mail p.socha@czd.pl 
señalan la necesidad de cambiar las recomendaciones para los lactantes sanos. Debe tenerse en cuenta que estas recomendaciones no rigen para la colestasis, que es un grupo de riesgo específico. La hepatopatía colestática debe ser diagnosticada precozmente y el tratamiento específico debe iniciarse lo antes posible. Numerosas hepatopatías colestáticas progresan hacia la insuficiencia hepática y requieren trasplante de hígado. La terapia nutricional parece ser una de la prioridades y puede considerarse como un medio para 'salir del paso' a la espera del trasplante. El estado nutricional es un factor pronóstico importante y niños con un estado nutricional más favorable presentan menos complicaciones y una mortalidad menor en el trasplante hepático [2]. Este es el motivo por el cual recientemente elaboramos y publicamos normas sobre la nutrición de lactantes con hepatopatía colestática [3].

Casi hemos olvidado las enfermedades relacionadas con deficiencias de nutrientes específicos. Recientemente, Samonte y cols. [4] describieron el caso de una niña que fue sometida a un trasplante hepático y tuvo que librar una enorme batalla frente a las complicaciones que se le presentaron, como insuficiencia renal en estadio terminal e insuficiencia hepática. Presentaba signos bucales clásicos de escorbuto, que se confundieron con toxicidad a la ciclosporina, alteraciones cutáneas que hacían pensar en una vasculitis y dolores óseos que se consideraron relacionados con osteodistrofia renal $\mathrm{u}$ osteoporosis inducida por corticosteroides. Por último, resultó evidente que la enfermedad estaba causada por una carencia de vitamina C. En la editorial de esta publicación indicamos que existen todavía situaciones en las que podemos hallar enfermedades básicas causadas por la carencia de un solo componente nutricional [5]. La hepatopatía grave con colestasis es un magnífico ejemplo de estos problemas nutricionales.

En este capítulo se presentan y comentan las causas de malnutrición, las necesidades de nutrientes específicos y un abordaje nutricional para niños con hepatopatía colestática.

\section{Causas de desnutrición}

La disminución del flujo de bilis conducente a la reducción de la concentración intraluminal de ácidos biliares parece el mecanismo patogenético principal responsable de la desnutrición. La colestasis lleva a una disminución significativa de la absorción de lípidos, y los triglicéridos de cadena larga requieren ácidos biliares para su utilización. Los lípidos contribuyen significativamente a la captación de energía y, en consecuencia, su malabsorción puede causar un balance energético negativo. También se documentan las elevadas necesidades de otros nutrientes, como proteína e hidrato de carbono. Numerosos estudios demuestran que la absorción y la utilización de lípidos y vitaminas liposolubles es un proceso en varias etapas: dispersión en emulsión lipídica, solubilización en micelas de sales biliares mixtas, movimiento a través de la capa acuosa no agitada adyacente a las microvellosidades, captación celular por las células de la mucosa intestinal, incorporación a los quilomicrones y secreción al interior de la linfa. En la colestasis, la absorción de algunas vitaminas puede disminuir considerablemente, como es el caso de la vitamina E o $\beta$-caroteno; otras vitaminas son mejor utilizadas y el aporte complementario de dosis elevadas puede equilibrar eficientemente la absorción deficiente. Se dispone de otros varios indicadores del riesgo de carencia, que deben ser objeto de evaluación; son los siguientes: ingestión baja de calorías, alimentación desequilibrada con bajo consumo de grasa y verduras, aumento del estado oxidativo debido a infecciones recurrentes y algunos otros procesos patológicos crónicos que complican la hepatopatía. Para estos grupos de alto riesgo se precisa una atención especial.

En la colestasis también se ha documentado un aumento del gasto de energía, que puede aumentar hasta el $40 \%$ o más para el peso corporal en la malnutrición, pero que es normal si se calcula para la edad $[6,7]$. La anorexia es un problema corriente de hepatopatía avanzada y puede atribuirse a organomegalia o ascitis. La hipertensión portal con derivaciones de aparición natural puede producir efectos centrales de toxinas no identificadas. A medida que hormonas del crecimiento importantes, como el factor de crecimiento de tipo insulínico I y sus proteínas ligadas, son producidas por el hígado en respuesta a la hormona del crecimiento circulante, la colestasis y la hipertensión portal pueden dar lugar a una respuesta anormal del eje del factor de crecimiento de tipo insulínico I. Estas anomalías no pueden ser corregidas por la hiperalimentación [8].

\section{¿Podemos aplicar un enfoque estándar en todas las enfermedades colestáticas?}

En general, la colestasis grave que progresa hacia la etapa terminal de la insuficiencia hepática causa una malabsorción significativa y exige un tratamiento nutricional intensivo. Sigue siendo difícil describir un abordaje 
estándar. Habitualmente, la lista de espera para trasplantes hepáticos constituye una indicación para la terapia nutricional con arreglo a protocolos estandarizados. No obstante, algunas enfermedades colestáticas progresan lentamente hasta la insuficiencia hepática o pueden no necesitar un trasplante hepático. La colestasis intrahepática familiar progresiva (CIFP) puede ser tratada con una intervención quirúrgica descrita como derivación biliar parcial. Aunque este tratamiento es capaz de detener la progresión a la insuficiencia hepática, la malabsorción de vitaminas o $\beta$-caroteno no se corrige. Esta enfermedad requiere una atención nutricional especial, dado que los niveles de vitamina $\mathrm{E}$ son extremadamente bajos en estos pacientes y no guardan relación directa con el grado de colestasis. En esta enfermedad se documenta frecuentemente una esteatorrea grave que no se relaciona con insuficiencia pancreática [9]. Las necesidades elevadas de aportes complementarios de vitamina E también se constatan en niños operados de atresia biliar, si bien se correlacionan perfectamente con el grado de colestasis.

Otro problema metabólico discutido en la colestasis es el nivel elevado de colesterol que se observa en el síndrome de Alagille. En algunos centros de hepatología se utiliza únicamente una dieta pobre en colesterol para tratar esta anomalía, mientras que en otros centros se recurre a la farmacoterapia para reducir los niveles de colesterol. En una publicación reciente en la que se compara el engrosamiento de la íntima y la media como indicador de aterogénesis en pacientes con CIFP (que presentan niveles bajos de colesterol) y síndrome de Alagille se demuestra que los pacientes con CIFP presentan un mayor riesgo de trastornos cardiovasculares que se complican con aterosclerosis. El engrosamiento de la íntima y la media y la rigidez parietal aumentan en pacientes con CIFP, pero no en pacientes con síndrome de Alagille. El colesterol total, el colesterol de lípidos de baja densidad, el colesterol de lípidos de alta densidad y la lipoproteína $\mathrm{X}$ aumentan notablemente en pacientes con síndrome de Alagille, mientras que en pacientes con CIFP los datos prominentes son un incremento de los triglicéridos y una reducción del colesterol de lípidos de alta densidad [10]. Por lo tanto, un enfoque nutricional no está destinado fundamentalmente a reducir los niveles de colesterol en el síndrome de Alagille.

La evaluación del estado nutricional puede causar también algunos problemas. La talla baja es una característica normal de la CIFP y no puede ser corregida mediante apoyo nutricional. Este es el motivo por el cual la talla no es un indicador satisfactorio del estado nutricional en la CIFP (fig. 1).

Tratamiento nutricional de los síndromes colestáticos en la infancia

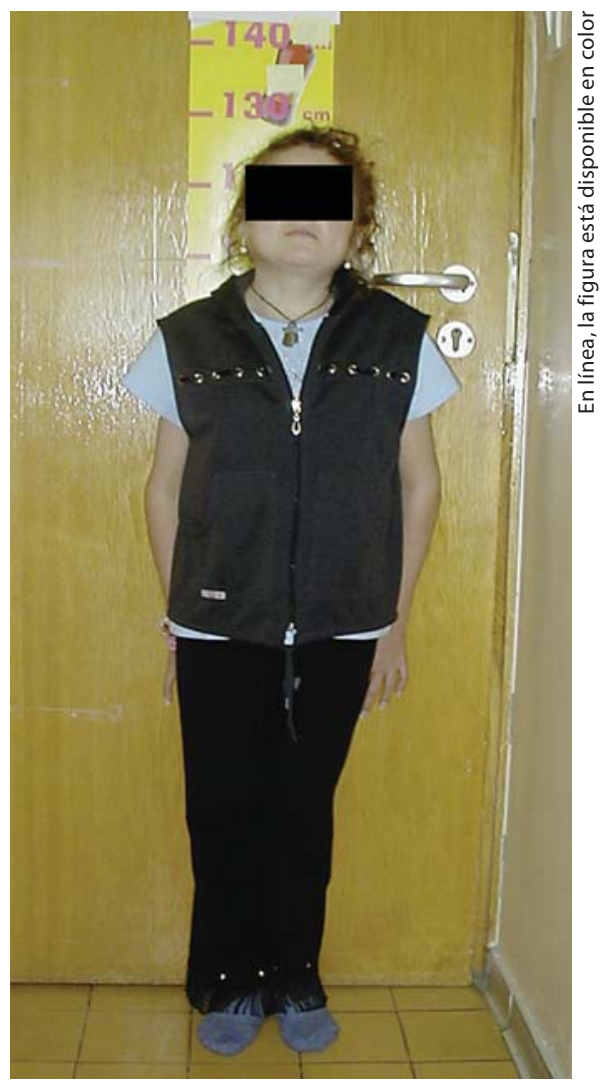

Fig. 1. Talla baja en una niña de 14 años afectada de CIFP.

\section{Diagnóstico de las deficiencias nutricionales}

La desnutrición de proteína y energía puede identificarse fácilmente si no se instaura un apoyo nutricional especial a partir del inicio temprano de la colestasis. Los niños pueden presentarse con extremidades delgadas y abdomen prominente. El peso corporal no se considera un buen indicador del estado nutricional, dado que es influido por anomalías del balance de líquidos [11, 12]. No obstante, si la ascitis es tratada eficientemente con diuréticos, los cambios en el peso corporal pueden utilizarse para le evaluación del estado nutricional. Puede recurrirse a otros parámetros antropométricos para evaluar el estado nutricional, como la circunferencia en la mitad del brazo y el grosor del pliegue cutáneo del tríceps. La talla puede afectarse si la desnutrición se prolonga. Este es el motivo por el cual la evaluación del estado nutricional puede ser complicada y deba tomarse también en cuenta la hepatopatía específica.

Los déficits de nutrientes específicos no pueden identificarse fácilmente y deben ser prevenidos lo antes posible. No obstante, en la práctica clínica aparecen todavía

Ann Nestlé [Esp] 2008;66:137-149 
síntomas clínicos de deficiencias de nutrientes, de los que el escorbuto o la hemorragia son sólo ejemplos. Este es el motivo por el cual es extremadamente importante detectar todos los signos de desnutrición muy precozmente. Tal como se ha indicado en las normas, todos los niños con hepatopatía colestática tienen que ser sometidos a una revisión nutricional completa con un plan de intervenciones y seguimientos documentado en sus historias clínicas [3]. Es necesario un registro de la ingestión de energía, proteínas, hidratos de carbono y lípidos, así como suplementos vitamínicos complementarios. Dietistas o nutricionstas deben participar en la evaluación y la planificación nutricionales. Sin embargo, la monitorización alimentaria minuciosa puede no ser suficiente para evitar la desnutrición y cambios significativos en la composición corporal. Algunas carencias pueden no acompañarse de desnutrición de proteína y energía y pueden ser detectadas precozmente únicamente mediante mediciones bioquímicas. Esto suscita las preguntas siguientes: ¿Deben evaluarse las vitaminas liposolubles en pacientes de grupos de alto riesgo? En segundo lugar, ¿deben aportarse sistemáticamente complementos vitamínicos e incrementar la ingestión de energía en estos pacientes de alto riesgo? La respuesta a la primera pregunta es afirmativa. Podemos describir con precisión los grupos de alto riesgo en los cuales la evaluación periódica de vitaminas podría ser beneficiosa en términos de diagnóstico precoz de la deficiencia. La respuesta a la segunda pregunta es más difícil. Aunque los aportes complementarios de vitamina $\mathrm{K}$ y vitamina $\mathrm{E}$ se consideran seguros, el exceso de vitaminas A y D en algunas circunstancias puede ser perjudicial y causar complicaciones graves.

Los pacientes ictéricos presentan osteoporosis más frecuentemente que los pacientes sin ictericia. La responsabilidad parece recaer en la carencia de vitamina D. La carencia de mineral óseo puede detectarse más precozmente por medio de densitometría (método no invasivo) que mediante la medición de los niveles séricos de $\mathrm{Ca}$, P y Mg en estos pacientes (tabla 1) [13].

\section{Ingestión de energía y carbohidratos}

Dado que las necesidades energéticas aumentan significativamente en la colestasis crónica, puede utilizarse una alimentación densa en energía para incrementar la ingestión calórica. En la práctica clínica, algunos servicios de hepatología utilizan leches concentradas para lactantes para niños colestáticos, en las que la densidad energética puede incrementarse hasta el 50\%. Pueden añadirse hidratos de carbono y proteínas a la alimentación normal. Los hidratos de carbono son la fuente principal de energía alimentaria, que contribuye en unos $2 / 3$ a la energía no procedente de proteínas. Los polímeros de cadena corta se utilizan actualmente como apoyo nutricional, dado que su carga osmótica no es suficientemente elevada para causar diarrea. Aunque también puede administrarse almidón, puede causar flatulencia y diarrea si no se digiere en el intestino delgado. En los pacientes deben monitorizarse los efectos secundarios cuando los polímeros de cadena corta se incrementan subsiguientemente durante unos pocos días. Si aparecen síntomas significativos de intolerancia, la dosis de hidrato de carbono no debe incrementarse ulteriormente. Aunque los hidratos de carbono se utilicen como fuente principal de energía, los lípidos pueden contribuir también a las mayores necesidades energéticas. Sin embargo, los hidratos de carbono suelen ser mejor aceptados por los pacientes, dado que tienen mejor sabor que los lípidos o las proteínas.

El cálculo de los hidratos de carbono agregados a la alimentación habitual debe basarse en las necesidades energéticas estimadas, que aumentan significativamente en la insuficiencia hepática de estadio terminal y en la atresia biliar con colestasis grave.

\section{Agua y electrolitos}

El requerimiento de líquidos es normal para el peso real, en caso de no ser necesaria una restricción debida a ascitis o edema. No deben aportarse complementos de sodio en cantidades elevadas para corregir la hiponatremia, dado que pueden causar retención de líquidos. En la lactancia suelen ser apropiadas una ingestión de sodio de $1 \mathrm{mmol} / \mathrm{kg} /$ día y una ingestión de potasio normal de unos $2 \mathrm{mmol} / \mathrm{kg} / \mathrm{día}$ [3]. Resulta difícil hallar el equilibrio apropiado entre la restricción de líquidos y la demanda elevada de energía. Este es el motivo por el cual debe utilizarse una alimentación densa en energía.

\section{Proteínas}

Los niños con crecimiento y peso normales necesitan habitualmente una ingestión normal de proteínas. Las necesidades de mantenimiento no parecen muy diferentes de las correspondientes a niños sanos. No obstante, la hepatopatía colestática avanzada con desnutrición debe ser tratada con terapia nutricional intensiva y una mayor ingestión de proteínas para sustentar las tasas elevadas de 
Tabla 1. Monitorización de las deficiencias de vitaminas y minerales

\begin{tabular}{|c|c|c|c|}
\hline $\begin{array}{l}\text { Elemento } \\
\text { nutricional }\end{array}$ & Signos clínicos de deficiencia & Signos de toxicidad & Monitorización bioquímica \\
\hline Vitamina A & $\begin{array}{l}\text { Habitualmente no se observa } \\
\text { piel seca, xeroftalmia y ceguera } \\
\text { nocturna }\end{array}$ & $\begin{array}{l}\text { Fibrosis hepática, hipercalcemia, pseudotumor } \\
\text { cerebral y lesiones óseas dolorosas }\end{array}$ & $\begin{array}{l}\text { Retinol sérico/PFR } \geq 0,8 \\
\text { Retinol sérico: } 250 \mathrm{a} \\
800 \mathrm{ng} / \mathrm{ml}\end{array}$ \\
\hline Vitamina D & $\begin{array}{l}\text { Osteopenia, fracturas óseas, } \\
\text { hipocalcemia, alteraciones } \\
\text { metafisarias, hipofosfatemia, } \\
\text { hipotonía muscular y raquitismo }\end{array}$ & Hipercalcemia y pseudotumor cerebral & $\begin{array}{l}\text { Niveles séricos de } 25-\mathrm{OH}-\mathrm{D} \\
>25 \mathrm{ng} / \mathrm{ml}\end{array}$ \\
\hline Vitamina $\mathrm{E}$ & $\begin{array}{l}\text { Neuropatía, edema, anemia, } \\
\text { lesiones cutáneas }\end{array}$ & $\begin{array}{l}\text { No documentados en niños colestáticos; dosis } \\
\text { extremadamente elevadas pueden afectar a la } \\
\text { quimiotaxis de los neutrófilos y la carboxila- } \\
\text { ción de las proteínas } \\
\text { Dependiente de vitamina K; seguridad del } \\
\text { aporte complementario puesta en entredicho } \\
\text { en adultos con respecto a la enfermedad } \\
\text { cardiovascular }\end{array}$ & $\begin{array}{l}\text { Vitamina E/lípidos totales } \\
\geq 0,6 \mathrm{mg} / \mathrm{g} \\
\alpha \text {-Tocoferol sérico } \\
3,8 \text { a } 16 \mathrm{mg} / \mathrm{l}\end{array}$ \\
\hline Vitamina $\mathrm{K}$ & Hemorragia & No documentados & $\begin{array}{l}\text { Tiempo de protrombina } \\
\text { PIVKA II }<3 \mathrm{ng} / \mathrm{ml}\end{array}$ \\
\hline $\begin{array}{l}\text { Vitaminas } \\
\text { hidrosolubles }\end{array}$ & $\begin{array}{l}\text { Habitualmente no aparecen; la } \\
\text { anemia puede estar relacionada } \\
\text { con deficiencia de ácido fólico y } \\
\text { vitamina } \mathrm{B}_{6} \text {; la neuropatía, con } \\
\text { deficiencia de vitaminas } \mathrm{B}_{1} \text { y } \mathrm{B}_{12} \text {; } \\
\text { el escorbuto, con deficiencia de } \\
\text { vitamina } \mathrm{C}\end{array}$ & No se observan con dosis normales & $\begin{array}{l}\text { Habitualmente no se realiza; } \\
\text { en grupos de alto riesgo } \\
\text { específicos, puede monitori- } \\
\text { zarse la vitamina C }\end{array}$ \\
\hline Hierro & Anemia, cambios de conducta & $\begin{array}{l}\text { La ferritina, el hierro y la hemoglobina en } \\
\text { suero no son muy confiables }\end{array}$ & \\
\hline $\begin{array}{l}\text { Minerales: } \\
\text { Calcio } \\
\text { Selenio } \\
\text { Cinc } \\
\text { Fosfato }\end{array}$ & $\begin{array}{l}\text { Osteoporosis, osteopenia, } \\
\text { fracturas óseas, retraso del } \\
\text { crecimiento, lesiones cutáneas, } \\
\text { anorexia, neutropenia }\end{array}$ & $\begin{array}{l}\text { No se observan con dosis normales; sin } \\
\text { embargo, el selenio administrado a dosis altas } \\
\text { puede ser tóxico }\end{array}$ & $\begin{array}{l}\text { Concentración sérica de calcio } \\
\text { y excreción urinaria de calcio/ } \\
\text { creatinina como rutinas de la- } \\
\text { boratorio; concentración sé- } \\
\text { rica de selenio y cinc en labo- } \\
\text { ratorios seleccionados }\end{array}$ \\
\hline
\end{tabular}

PFR = Proteína que fija retinol.

recuperación del crecimiento. Un cociente proteínas/ energía del $10 \%$, utilizado para rehabilitar a niños desnutridos, es en general suficiente para asegurar una recuperación rápida del crecimiento si sólo se cubren las necesidades energéticas [14]. Las necesidades específicas en la colestasis no se han descrito claramente. Los lactantes con hepatopatía colestática grave parecen requerir una ingestión de proteínas en torno a $2-3 \mathrm{~g} / \mathrm{kg} / \mathrm{dí}$. Las necesidades son menores en la colestasis de la infancia, así como en niños y lactantes sanos. Las infecciones que se observan frecuentemente en la atresia biliar (como episo- dios de colangitis) pueden incrementar adicionalmente las necesidades de energía y proteínas. En niños, la proteolisis aumenta notablemente durante las infecciones y puede aparecer un balance proteínico negativo [15]. La restricción de proteínas puede considerarse únicamente en presencia de encefalopatía, como es el caso de la insuficiencia hepática y las derivaciones portosistémicas relacionadas con hipertensión portal. La hiperamonemia no justifica la restricción de proteínas en ausencia de encefalopatía. 
En lactantes y niños con hepatopatía colestática se suministra sistemáticamente una dieta polimérica. Los hidrolizados proteínicos pueden considerarse en la nutrición enteral o transitoriamente en la desnutrición grave. El sabor de los alimentos debe también tomarse en cuenta como factor importante, dado que la mala aceptabilidad puede disminuir ulteriormente la ingestión de energía. Por lo tanto, los hidrolizados pueden utilizarse únicamente en situaciones clínicas muy específicas.

Recientemente, se han investigado los aminoácidos de cadena ramificada en modelos animales y en situaciones clínicas de atresia biliar [16, 17]. En niños colestáticos mejoraron la ganancia de peso, la masa muscular, el balance nitrogenado y también dieron lugar a un incremento del potasio corporal total, la circunferencia en la mitad del brazo y el espesor del pliegue cutáneo subescapular.

Las normas recientes proponen un producto ideal que incluiría proteínas en forma de $3 \mathrm{~g} / \mathrm{kg} /$ día de proteínas del suero de leche, representando aproximadamente 2,6 g/ $100 \mathrm{ml}$ de leche reconstituida para lactantes, enriquecida con aminoácidos de cadena ramificada hasta el $10 \%$, tratando de este modo la hiperamonemia en lugar de reducir la ingestión de proteínas [3].

\section{Lípidos}

El suministro alimentario y el metabolismo de los lípidos en la primera infancia tienen gran importancia para el crecimiento, la composición corporal y el desarrollo de los niños. Las selecciones de los lípidos alimentarios durante los primeros años de vida tienen gran importancia práctica para el crecimiento y el desarrollo, no sólo en lactantes y niños sanos, sino incluso todavía más en niños con trastornos hepáticos. Los lípidos constituyen la fuente principal de energía suministrada por la leche humana. El suministro de lípidos alimentarios es también importante para la provisión de vitaminas liposolubles y ácidos grasos poliinsaturados (AGPI) esenciales [18].

\section{Los lípidos como fuente de energía}

La absorción de lípidos disminuye notablemente en la colestasis, lo que lleva a reducción de la ingesta de energía. Por lo tanto, es importante equilibrar las pérdidas de energía con un suministro energético extra. Aunque los hidratos de carbono pueden incrementar la densidad energética de la alimentación hasta cierta medida, no son suficientes para cubrir todas las necesidades energéticas. Aunque el lípido es menos aceptable, las demás características siguientes de este macronutriente lo convierten en un complemento alimentario valioso: densidad energética elevada, osmolaridad baja y contenido en AGPI esenciales. La esteatorrea puede limitar el uso de lípidos en el apoyo nutricional, aunque en un niño con esteatorrea, el crecimiento puede mejorar si los lípidos, administrados en cantidades más elevadas, incrementan la ingesta de grasa [19]. Los triglicéridos de cadena media (TCM) pueden utilizarse opcionalmente como aporte complementario lipídico o como leche para lactantes enriquecida en TCM. Los TCM no proporcionan el mismo balance energético que los triglicéridos de cadena larga (TCL), pero se utilizan con éxito en el tratamiento de la malabsorción de grasa porque poseen una hidrosolubilidad elevada y son escindidos rápidamente por las lipasas [20]. No obstante, debido a la longitud más corta de las cadenas de sus ácidos grasos, su contenido en energía (por gramo de grasa) es alrededor del 16\% menor que el de los TCL. Los TCM son oxidados rápidamente y presentan un elevado efecto termógeno. Aunque alcanzan el hígado directamente a través del flujo portal sin necesidad de formación de micelas mixtas, son también cetógenos [21]. Cantidades mayores de TCM alimentarios son metabolizadas en varios tejidos por un mecanismo que depende de la carnitina y pueden incrementar la necesidad de carnitina [22]. Se puede estimar que la proporción de TCM del 30 al 50\% de los lípidos totales es óptima para el tratamiento nutricional de la colestasis [23, 24]. Sin embargo, es difícil especificar el contenido en grasa alimentaria y el cociente entre TCM y TCL ideales. Los datos limitados disponibles indican una mejor solubilización de las grasas y un mejor crecimiento de los lactantes colestáticos alimentados con el 30 ó el 70\% de TCM frente a una mezcla del 50/50\% de TCM/TCL [25]. Se describió la deficiencia de ácidos grasos esenciales con el empleo de un contenido en TCM muy elevado en la alimentación, que no había sido complementada suficientemente con AGPI [23]. La mayoría de las leches para lactantes con TCM presentan un cociente TCM/TCL de 1/1 aproximadamente y son suplementadas con ácidos grasos esenciales. Las leches para niños, ricas en TCM, pueden contener mayores cantidades de TCM (del 75\% al >80\% de lípidos; por ejemplo, Caprilon y Portagen), a pesar de que son suplementadas con ácidos grasos esenciales. Se precisa una atención especial si se utiliza como aporte complementario alimentario aceite de TCM puro, situación en la que se requiere una evaluación alimentaria detallada. Los aceites vegetales ricos en AGPI deben utilizarse también para sustentar la ingestión adecuada de ácidos grasos esenciales. La ingestión de lípidos y el cociente TCM debe valorarse frente a la ganancia de peso y el crecimiento 
óptimo y la intolerancia de los nutrientes. Los lípidos deben utilizarse para incrementar la densidad energética y una proporción por lo menos similar a la de niños sanos de edades equiparables (del 30 al 50\%) parece razonable [3].

\section{Ácidos grasos poliinsaturados}

Los AGPI de cadena larga (PCL, AGPI con cadena carbonada de longitud $>18$ ), como el ácido araquinódico (AA) y el ácido docosahexaenoico (ADH) son importantes para el crecimiento humano temprano y el desarrollo de tejidos ricos en membranas, como el cerebro y la retina [26]. Los AGPI son también precursores de los eicosanoides, cuyos importantes papeles biológicos como mediadores de las funciones inmunitaria y vascular y la agregación plaquetar convierten en un problema la deficiencia de AGPI (especialmente de la serie n-6). Los ácidos grasos esenciales clásicos, ácido linoléico (18:2n-6) y ácido $\alpha$-linolénico (18:3n-3), deben ser suministrados en la alimentación, tras de lo cual son elongados en el hígado y el cerebro para obtener PCL [18]. Los lactantes alimentados con leche materna reciben con los lípidos de la misma cantidades apreciables de AA y ADH preformados [27]. En contraste con los lactantes alimentados con leche humana, los que reciben fórmulas lácteas convencionales basadas en aceites vegetales no obtienen cantidades apreciables de PCL con su alimentación y dependen de la utilización de depósitos corporales o la síntesis endógena de precursores de ácidos grasos esenciales para la provisión de PCL necesaria para los tejidos en fase de crecimiento. Koletzko y cols. [28] estimaron las tasas de conversión del ácido linoleico con una técnica de isótopos estables y hallaron que la contribución de la síntesis de AA a la reserva plasmática total de AA sólo es de alrededor del $6 \%$ al día. Sin embargo, para llegar a cualquier conclusión sobre los beneficios del suministro de PCL en la lactancia, se precisan datos fehacientes de ensayos clínicos aleatorizados. En una revisión Cochrane de 2004 se revisaron publicaciones sobre el aporte complementario de PCL en lactantes a término hasta 2001, incluyendo estudios sobre la agudeza visual, así como la función cognitiva y el desarrollo psicomotor [29]. Se aplicaron métodos diferentes para medir los efectos funcionales de complementos, que generan dificultades para establecer conclusiones. Los autores llegaron a la conclusión de que es posible un efecto beneficioso del suministro de PCL sobre el procesamiento de información; sin embargo, se ha sugerido la necesidad de estudios más extensos durante periodos más prolongados para investigar adicionalmente esta cuestión. En una revisión crítica de las publicaciones disponibles, Uauy y cols. [30] indicaron que en algunos estudios en los que no se hallaron ventajas de los PCL, o bien se habían utilizado concentraciones bajas del ADH o no se había incluido una evaluación del cumplimiento.

En publicaciones recientes que no están incluidas en la revisión Cochrane se estudiaron suministros de PCL más tardíos en lactantes tras el destete de la alimentación con leche materna en los primeros meses de vida. Birch y cols. [31] estudiaron a lactantes que habían sido destetados después de unos 6 meses desde el suministro de leche materna hasta el suministro de leches para lactantes con o sin PCL, hallando una menor agudeza visual al cabo de 17,26 y 52 semanas de edad en los lactantes que no recibieron PCL. Análogamente, Hoffman y cols. [32] observaron una maduración más rápida de los potenciales visuales evocados al año de edad en lactantes que recibieron aportes complementarios a partir de los 4 meses. Por otra parte, se demostró recientemente que el aporte complementario temprano de PCL puede influir tanto sobre la presencia de tipos específicos de células inmunitarias como sobre su función [33].

Dado que la estimación de los requerimientos de AGPI y PCL no es fácil para lactantes y niños sanos, incluso es más difícil impartir recomendaciones para niños con colestasis. Sin embargo, es preciso ser consciente del riesgo de la carencia de AGPI; al respecto, se describieron algunos casos clínicos con los síntomas dermatológicos clásicos de la carencia de ácidos grasos esenciales [34]. Hay varios factores que contribuyen a la carencia de AGPI y PCL; entre ellos destacan la ingestión escasa de AGPI, la malabsorción y el trastorno metabólico de los AGPI para formar derivados de cadena larga. Describimos la carencia de PCL y AGPI expresada por los niveles plasmáticos de fosfolípidos en lugar del contenido adecuado en AGPI en la alimentación de lactantes colestáticos [35]. La ingestión extra de AGPI puede mejorar el estado de los ácidos grasos (por ejemplo, aceite de soja o aceite de colza) y, en la lactancia, parece que debe sobrepasar significativamente el $10 \%$ de la energía total.

$\mathrm{El}$ aporte complementario de ácidos grasos esenciales puede no ser suficiente. Fuimos capaces de demostrar un deterioro creciente de la conversión de AGPI hepáticos en PCL con un anticipo de la gravedad de la hepatopatía; por lo tanto, la colestasis grave es un factor de riesgo significativo de la carencia de PCL, incluso en presencia de un suministro de ácidos grasos esenciales [36]. Puede requerirse el aporte complementario de PCL basándose en productos alimenticios como la yema de huevo (rica en AA) o el aceite de pescado (rico en $\mathrm{ADH}$ ). Los lactantes 
pueden recibir leches convencionales para lactantes, complementadas con PCL, disponibles para lactantes a término y prematuros sanos, o aportes complementarios alimentarios. En la carencia de los AGPI no sólo puede desempeñar un papel su ingestión escasa sino también el deterioro de su metabolismo y la reducción de su absorción. Los ácidos grasos insaturados pueden experimentar una peroxidación lipídica, cuyo incremento se observa en la colestasis causada por el deterioro de la defensa antioxidante. Incluso si registramos niveles elevados de peroxido lipídico y concentraciones bajas de vitamina E, no seremos capaces de demostrar ninguna relación directa entre los AGPI y los peróxidos lipídicos [37, 38]. Puede aparecer una deficiencia de PCL intensa en la hepatopatía colestática avanzada, que puede ser difícil de corregir. Se ha documentado que incluso un año tras el trasplante hepático, el estado de los PCL no ha llegado a invertirse por completo [39]. Por el momento no se dispone de estudios capaces de demostrar los efectos funcionales del aporte complementario de PCL en niños con colestasis, por lo que no podemos facilitar recomendaciones sólidas sobre el aporte complementario de AGPI y PCL. En la práctica clínica está justificado el uso de aceites vegetales ricos en AGPI y/o yema de huevo como complementos alimentarios para incrementar la densidad energética y la ingestión de AGPI. En lactantes con colestasis que reciben regularmente leches para lactantes y no necesitan una alimentación enriquecida con TCM, pueden utilizarse las fórmulas para lactantes complementadas con PCL [3].

\section{Vitaminas}

La malabsorción de las vitaminas liposolubles parece ser el problema nutricional principal, muy específico de la colestasis. Algunos otros factores pueden contribuir también a los niveles bajos de vitaminas liposolubles, como la utilización de vitamina E debido al incremento de la formación de radicales libres. Mientras que algunas vitaminas liposolubles pueden ser complementadas eficientemente (vitaminas A, D y K), el aporte complementario de vitamina $\mathrm{E}$ puede causar problemas significativos. En la hepatopatía colestática grave, la vía oral para el aporte complementario de vitaminas puede no ser suficiente y, por tanto, deben considerarse las formulaciones parenterales.

\section{Deficiencia de vitamina $A$}

La deficiencia de vitamina A se manifiesta con piel seca, xeroftalmia y ceguera nocturna. Se recurre a menu- do a los niveles séricos para estimar el estado de vitamina A, el cual, sin embargo, no queda correctamente reflejado por este procedimiento. Un indicador útil del estado de vitamina $\mathrm{A}$ es el cociente molar de retinol/proteína que fija retinol en el plasma [calculado como retinol sérico $(\mathrm{ng} / \mathrm{dl}) /$ proteína que fija retinol en el suero $(\mathrm{mg} / \mathrm{dl}) \times$ $0,0734]$. Los cocientes superiores a 0,8 indican un estado normal de vitamina A. El metabolismo hepático de la vitamina A suele estar conservado en los pacientes colestáticos $[40,41]$. Los cocientes superiores pueden asociarse a toxicidad, destacando al respecto la fibrosis hepática, la hipercalcemia, el pseudotumor cerebral y las lesiones óseas dolorosas. La toxicidad causada por la vitamina A puede documentarse satisfactoriamente a partir de los niveles plasmáticos de los ésteres de vitamina $A$. Dado que la vitamina A puede ser tóxica, se puede considerar un aporte complementario de $\beta$-caroteno como precursor de vitamina A. También se trata de una sustancia liposoluble que participa en la protección de las membranas frente al daño causado por los radicales libres, de forma complementaria a la acción de la vitamina $\mathrm{E}$, y puede utilizarse fiablemente como aporte complementario en pacientes con hepatopatías, al contrario que el caso del aporte complementario de vitamina A. Existen pruebas convincentes de estudios in vitro de que el $\beta$-caroteno es un antioxidante potente que puede aliviar el efecto tóxico de los ácidos biliares sobre los hepatocitos [42]. Asimismo, se ha descrito una deficiencia de $\beta$-caroteno en adultos con colestasis prolongada [43]. Hemos comunicado recientemente niveles bajos de $\beta$-caroteno en niños con colestasis, que podrían estar relacionados con la absorción deficiente de esa sustancia en el intestino (datos listos para su publicación).

\section{Deficiencia de vitamina $D$}

La deficiencia de vitamina D puede presentarse también fácilmente en la colestasis prolongada. Los lactantes alimentados con leche materna están en un mayor riesgo, dado que dicha leche contiene cantidades bajas de vitamina D. Síntomas de deficiencia extrema de vitamina D se observan en raras ocasiones, como hipocalcemia, hipofosfatemia, hipotonía muscular y raquitismo. En la hepatopatía grave, no sólo es responsable de la mala disponibilidad de dicha vitamina la absorción deficiente de vitamina $\mathrm{D}$, sino que también el deterioro de su metabolismo en el hígado puede desempeñar un papel significativo. Este es el motivo por el cual para corregir una carencia no se utiliza vitamina D sino su metabolito, 25-hidroxicolecalciferol (25-OH-D). Debe tenerse en cuenta que el aporte complementario de vitamina $\mathrm{D}$ en solitario 
no es eficaz y que, al mismo tiempo, deben administrarse calcio y fósforo. No obstante, el tratamiento con vitamina D no siempre es satisfactorio en la mejora del trastorno óseo [44]. Aunque la exposición a la luz solar puede ser de cierta ayuda, no basta. El mejor indicador del estado de vitamina $\mathrm{D}$ en la colestasis es la concentración sérica de $25-\mathrm{OH}-\mathrm{D}$; se considera que niveles de $20 \mathrm{ng} / \mathrm{ml}$ o menos son extremadamente bajos y se discuten los valores óptimos en individuos sanos. Las concentraciones que superan significativamente los $25 \mathrm{ng} / \mathrm{ml}$ pueden considerarse deseables. También puede utilizarse el indicador simple de hipocalcemia; el cociente calcio/creatinina en la orina debe ser $<0,25$. No obstante, no hay ninguna correlación directa entre la gravedad de la osteopenia y los niveles séricos de $25(\mathrm{OH})$-vitamina $\mathrm{D}$ y $1,25(\mathrm{OH}) 2$-vitamina $\mathrm{D}$ ni en lactantes ni en niños mayores con colestasis. La disminución de la mineralización ósea en la colestasis infantil es un proceso patológico que se inicia precozmente en la lactancia, empeora rápidamente a medida que aumenta la edad y la disfunción hepática y permanece relativamente estable en niños con hepatopatía más estable [44]. La toxicidad por vitamina $\mathrm{D}$ se describe en raras ocasiones e incluye hipercalcemia y pseudotumor cerebral. El trasplante hepático parece corregir la osteopenia y la deficiencia de vitamina D. En lactantes y niños menores de 2 años con colestasis crónica, el contenido en mineral óseo se normaliza aproximadamente a los 11 meses tras el trasplante hepático ortotópico. Esta normalización viene precedida por un periodo persistente de niveles séricos normales de 25-OH-D [45].

\section{Deficiencia de vitamina $E$}

La deficiencia de vitamina $E$ parece constituir un problema muy importante en la colestasis prolongada, difícil de corregir en algunas enfermedades colestáticas. La deficiencia notable de vitamina $\mathrm{E}$ en niños con colestasis crónica causa degeneración neurológica. El papel más importante de la vitamina $\mathrm{E}$ es su función antioxidante como depurador de radicales libres de oxígeno. La disponibilidad insuficiente de esta vitamina puede predisponer a la peroxidación, incluyendo la de los ácidos grasos insaturados, que perturba la función de las membranas celulares de los sistemas nerviosos central y periférico. Las consecuencias de la avitaminosis E son especialmente serias en niños. Las alteraciones neurológicas inicialmente sutiles pueden, al cabo de algunos años, llevar a un daño irreversible. Entre los signos neurológicos relacionados con la deficiencia de vitamina E destacan habitualmente los siguientes: neuropatía periférica, oftalmoplejia, ataxia, deterioro de la sensación vibratoria y lesiones degenerativas de la retina. Los primeros signos, en forma de desaparición de los reflejos tendinosos profundos, pueden manifestarse con relativa precocidad. Las lesiones del sistema nervioso son similares a las observadas en otras enfermedades por deficiencia de vitamina E, como la abetalipoproteinemia, la enfermedad de Anderson y la deficiencia familiar, selectiva y aislada de vitamina E [46-48].

En numerosos niños con colestasis no es posible obtener una mejora del estado de vitamina $\mathrm{E}$ con preparados liposolubles por vía oral, lo que obliga a recurrir a inyecciones intramusculares dolorosas. Sokol y cols. [49-51] comunicaron el efecto beneficioso de un nuevo preparado de vitamina $\mathrm{E}$ hidrosoluble, por vía oral, que corrige los niveles séricos de $\alpha$-tocoferil y mejora los trastornos neurológicos existentes en niños con colestasis. El TPGS (d- $\alpha$-tocoferil polietilenglicol 1.000 succinato, Eastman Chemical Products Inc., Kingsport, Tenn., EE.UU. u Orphan Europe, París, Francia) es un éster de ácido succínico de vitamina $\mathrm{E}$ hidrosoluble ligado al polietilenglicol 1.000 , que puede formar micelas sin la intervención de ácidos biliares. Nosotros también informamos sobre los efectos del tratamiento con TPGS, que normalizó rápidamente los niveles séricos de vitamina E, pero no mejoró el aumento de la peroxidación lipídica ni el estado deficiente de AGPI [37]. Puede haber una relación con la administración concomitante de otras vitaminas liposolubles en pacientes con colestasis [52]. La monitorización de la vitamina $\mathrm{E}$ se basa en el cociente vitamina E/lípidos totales (normal $>0,8 \mathrm{mg} / \mathrm{g}$, pero con vitamina E plasmática $<30 \mu \mathrm{g} / \mathrm{ml})$. La toxicidad es inusitada.

\section{Deficiencia de vitamina $K$}

La deficiencia de vitamina K puede ser un signo precoz de malabsorción de grasa en la colestasis [1], que resulta en una enfermedad hemorrágica. De los registros nacionales de atresias biliares de Holanda y Dinamarca se infieren conclusiones importantes. Una dosis diaria de 25 $\mu \mathrm{g}$ de vitamina $\mathrm{K}$ fue incapaz de evitar hemorragias en lactantes aparentemente sanos, con colestasis debida a una atresia biliar no diagnosticada. Un miligramo de profilaxis oral semanal confirió una protección significativamente superior a estos lactantes y su eficacia fue similar a la de $2 \mathrm{mg}$ de profilaxis intramuscular en el nacimiento [53]. En la práctica clínica se utiliza una dosis elevada, por vía oral, de vitamina $\mathrm{K}$ cada semana o cada tres días para prevenir las hemorragias. Para el tratamiento de un tiempo de protrombina significativo, la intensificación de la administración intravenosa de vitamina K es la mejor opción. El Konakion MM (Roche) parece ser una 
Tabla 2. Vitaminas liposolubles en el tratamiento de niños colestáticos

\begin{tabular}{|c|c|c|}
\hline $\begin{array}{l}\text { Vitamina } \\
\text { liposoluble }\end{array}$ & Necesidad diaria & $\begin{array}{l}\text { Vía de } \\
\text { administración }\end{array}$ \\
\hline Vitamina A & $\begin{array}{l}\text { <10 kg: } 5.000 \mathrm{UI} \\
\text { >10 kg: } 10.000 \mathrm{UI} \\
\text { IM: } 50.000 \mathrm{UI}\end{array}$ & Oral \\
\hline Vitamina D & $\begin{array}{l}25-\mathrm{OH}-\mathrm{D}: 2-5 \mu \mathrm{g} / \mathrm{kg} \\
\text { IM: } 30.000 \text { UI } \\
1 \text { a } 3 \text { mensualmente }\end{array}$ & Oral/IM \\
\hline Vitamina E & $\begin{array}{l}\text { TPGS } 25 \text { UI/kg } \\
\text { IM: } 10 \text { mg/kg (máx. } 200 \text { mg) } \\
\text { Cada } 3 \text { semanas }\end{array}$ & Oral \\
\hline Vitamina $\mathrm{K}$ & $\begin{array}{l}2 \mathrm{mg} / \text { día } \\
\text { Semanalmente } \\
5-10 \mathrm{~kg}: 5 \mathrm{mg} \\
\text { >10 kg: } 10 \mathrm{mg} \\
\text { IM: } 5-10 \mathrm{mg} \text { cada } 2 \text { semanas }\end{array}$ & Oral \\
\hline
\end{tabular}

Con arreglo a Baker y cols. [3]. IM = Intramuscular; máx. = máximo.

formulación (compuesto de vitamina K solubilizado en glicocolato y lecitina) que corrige eficiente y fiablemente la deficiencia de vitamina $\mathrm{K}$ [54].

La deficiencia de vitamina $\mathrm{K}$ puede detectarse por el índice internacional normalizado (incremento del tiempo de protrombina), que es corregido por la administración parenteral de vitamina K. La PIVKA II (proteína inducida en ausencia de vitamina K), medida por el análisis ELISA, parece ser un mejor indicador que el tiempo de protrombina (un PIVKA II $>3 \mathrm{ng} / \mathrm{ml}$ refleja una carencia de vitamina $\mathrm{K}$ ); sin embargo, no se halla corrientemente disponible.

Deben aportarse también complementos de vitaminas hidrosolubles, dado que la malabsorción de nutrientes puede causar también deficiencias de vitaminas hidrosolubles. Por otra parte, los antioxidantes pueden disminuir a medida que son utilizados en la protección frente a la formación elevada de peróxidos. La carencia de un solo nutriente, como la vitamina $\mathrm{C}$, puede ser incluso más intensa si se combina con niveles bajos de otros antioxidantes (por ejemplo, vitamina E). En la colestasis crónica se recomienda utilizar el doble de la ingestión diaria recomendada de vitaminas hidrosolubles [3]; sin embargo, en algunas circunstancias se precisan dosis muy superiores de vitaminas concretas [4]. Se han documentado niveles bajos de minerales y oligoelementos en niños con colestasis. Deben administrarse aportes complementarios de selenio, cinc, calcio y magnesio según los niveles plasmáticos [3]. El selenio y el cinc son antioxidantes importantes. El hierro puede incrementar la agresión oxidativa y la fibrogénesis en pacientes con hepatopatía [55]. Por lo tanto, el hierro no debe ser aportado de forma regular. La deficiencia de hierro no parece un problema corriente en la colestasis. El trasplante hepático parece corregir la mayoría de las deficiencias y el estado antioxidante y restaurar dichas capacidades excepto para el glutatión (tabla 2) [56].

\section{Vías de administración oral, enteral y parenteral}

La vía oral es preferible únicamente si puede asegurarse un suministro suficiente de energía y nutrientes. Para la alimentación oral deben utilizarse preparados comerciales y establecer una dieta bien equilibrada bajo el control de un especialista en nutrición. Los alimentos deben ser aceptables. Para incrementar la ingesta energética pueden utilizarse preparados densos en energía.

En la hepatopatía avanzada, especialmente si es necesaria una recuperación rápida del crecimiento antes del trasplante hepático, la alimentación por sonda nasogástrica puede ser la mejor opción. La alimentación por embolada no siempre puede ser tolerada. Un ritmo de infusión constante, logrado mediante bombeo durante un periodo de hasta $20 \mathrm{~h}$ al día, puede resolver muchos de los problemas que aparecen con la alimentación por sonda, como el vómito. La alimentación por sonda nasogástrica nocturna es otra opción: se permite al niño que coma a voluntad durante el día y recibe energía extra cuando duerme. La preparación diaria de las soluciones requiere la participación de especialistas en nutrición. El riesgo de contaminación bacteriana de la alimentación debe constituir una alerta. La gastrostomía endoscópica percutánea no es un modo corriente de suministrar nutrientes debido al riesgo de hemorragia varicial.

La nutrición parenteral se necesita en un porcentaje muy reducido de pacientes, dado que habitualmente está conservada la función absortiva del tubo digestivo. El vómito, la flatulencia, la diarrea y otras complicaciones pueden causar problemas graves en la nutrición oral y enteral. Si la ingestión de energía es demasiado escasa para cubrir las necesidades, la nutrición parenteral parcial puede mejorar el estado nutricional. Sin embargo, la seguridad de este método de terapia nutricional está sólidamente relacionada con la experiencia del centro clínico. Las normas de nutrición parenteral en la colestasis son similares a las que rigen para otras indicaciones. 
Se utilizan soluciones estándar de aminoácidos; no ha sido documentado el beneficio de los aminoácidos de cadena ramificada [57]. También los lípidos son bien tolerados. En niños colestáticos, evaluamos la seguridad de infusiones lipídicas de TCL y TCM/TCL en nutrición parenteral a corto plazo y hallamos una favorable tolerabilidad metabólica de ambas emulsiones infundidas por vía intravenosa en lactantes con colestasis. Dado que el ADH parece desempeñar un papel importante en el desarrollo del cerebro, las emulsiones de TCL pueden ser la fuente óptima de AGPI (contienen más AGPI que las emulsiones de TCM/TCL) para lactantes con colestasis crónica siempre que se administren a corto plazo. Nuestros resultados no revelan ningún riesgo de incremento de peroxidación lipídica con las infusiones de lípidos. Las concentraciones plasmáticas de lípidos no cambiaron con ninguna de las emulsiones lipídicas utilizadas [58].

Guimber y cols. [59] también comunicaron la seguridad de la nutrición parenteral administrada en niños colestáticos. En 7 niños con hepatopatía observaron un incremento de las puntuaciones $\mathrm{Z}$ del peso para la edad y del peso para la talla, sin cambio significativo en los parámetros de función hepática sintética. Es probable que el incremento significativo de la bilirrubina guardase relación con la evolución natural de la hepatopatía o que pudiera explicarse parcialmente por la nutrición parenteral. Incluso si la nutrición parenteral prolongada es un factor de riesgo de colestasis en pacientes que no presentan hepatopatía primaria, este método de terapia nutricional puede utilizarse para 'salir del paso' a la espera del trasplante hepático.

\section{Conclusiones}

La hepatopatía colestática merece atención especial con respecto a la nutrición y el apoyo nutricional. Debe evaluarse el estado nutricional y determinarse las necesi-

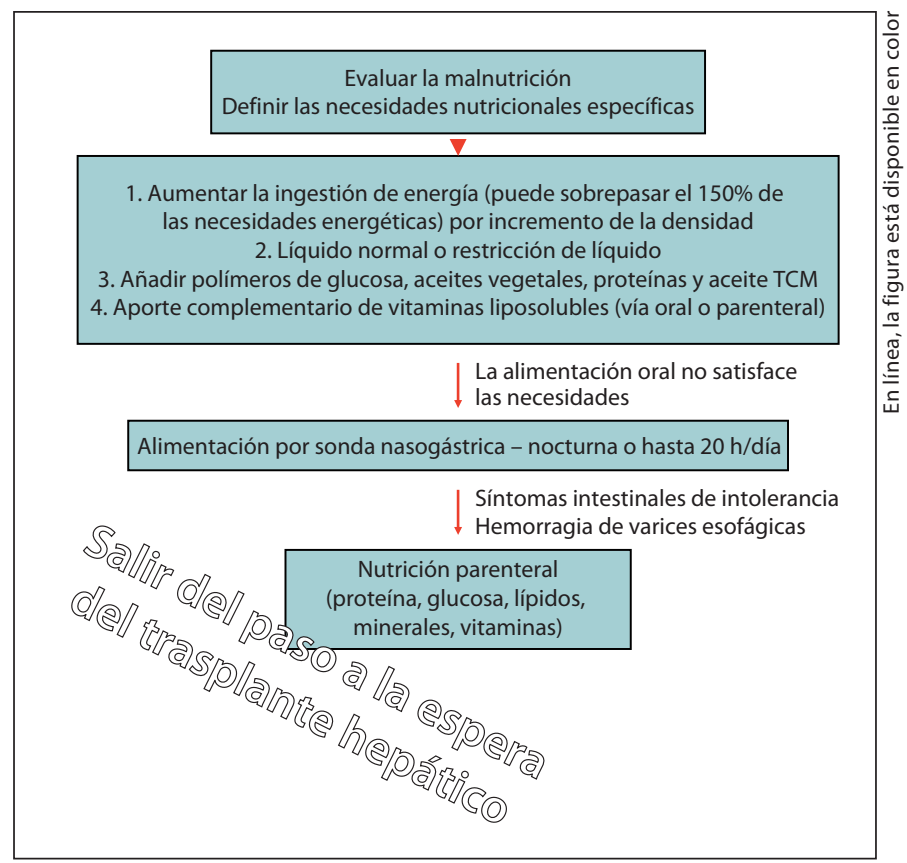

Fig. 2. Algoritmo de alimentación para niños con hepatopatía colestática.

dades nutricionales específicas antes de que pueda iniciarse la terapia nutricional. El principio básico del tratamiento nutricional consiste en corregir el estado nutricional con suministro adicional de proteínas energéticas, hidratos de carbono, lípidos y vitaminas liposolubles. A medida de que la hepatopatía progrese hacia la insuficiencia hepática en numerosas enfermedades colestáticas crónicas, la terapia nutricional debe considerarse a menudo, para 'salir del paso' a la espera del trasplante hepático, con objeto de mejorar el pronóstico. Por lo tanto, el apoyo nutricional invasivo está justificado en la hepatopatía grave; habitualmente comprende la nutrición por sonda nasogástrica nocturna o incluso la nutrición parenteral (fig. 2).

\section{Bibliografía}

1 Ijland MM, Pereira RR, Cornelissen EAM: Incidence of late vitamin $\mathrm{K}$ deficiency bleeding in newborns in the Netherlands in 2005: evaluation of the current guideline. Eur J Pediatr 2008; 167:165-169.

$\checkmark 2$ Chin SE, Shepherd RW, Thomas BJ, et al: The nature of malnutrition in children with endstage liver disease awaiting orthotopic liver transplantation. Am J Clin Nutr 1992;56: 164-168.
-3 Baker A, Stevenson R, Dhawan A, et al: Guidelines for nutritional care for infants with cholestatic liver disease before liver transplantation. Pediatr Transplant 2007;11: 825-834.

4 Samonte V, Sherman P, Taylor G, et al: Scurvy diagnosed in a paediatric liver transplant patient awaiting combined kidney and liver transplantation. Pediatr Transplant 2008;12: 363-367.
5 Socha P, Litwin M: Editorial to: Scurvy diagnosed in a pediatric liver transplant awaiting combined kidney and liver transplantation. Pediatr Transplant 2008;12:257-260.

6 6 Heymsfield SB, Waki M, Reinus J: Are patients with chronic liver disease hypermetabolic? Hepatology 1990;11:502-505. 
7 Greer R, Lehnert M, Lewindon P, et al: Body composition and components of energy expenditure in children with end-stage liver disease. J Pediatr Gastroenterol Nutr 2003; 36:358-363.

8 Holt RI, Miell JP, Jones JS, et al: Nasogastric feeding enhances nutritional status in paediatric liver disease but does not alter circulating levels of IGF-I and IGF-binding proteins. Clin Endocrinol 2000;52:217-224.

-9 Walkowiak J, Jankowska I, Pawlowska J, et al: Exocrine pancreatic function in children with progressive familial intrahepatic cholestasis type 2. J Pediatr Gastroenterol Nutr 2006;42:416-418.

10 Nagasaka H, Yorifuji T, Egawa H, et al: Evaluation of risk for atherosclerosis in Alagille syndrome and progressive familial intrahepatic cholestasis: two congenital cholestatic diseases with different lipoprotein metabolisms. J Pediatr 2005;146:329-335.

-11 Sokol RJ, Stall C: Anthropometric evaluation of children with chronic liver disease. Am J Clin Nutr 1990;52:203-208.

-12 Trocki O, Wotton MJ, Cleghorn GJ, Shepherd RW: Value of total body potassium in assessing the nutritional status of children with end-stage liver disease. Ann NY Acad Sci 2000;904:400-405.

13 Chongsrisawat V, Ruttanamongkol P, Chaiwatanarat $\mathrm{T}$, et al: Bone density and 25-hydroxyvitamin D level in extrahepatic biliary atresia. Pediatr Surg Int 2001;17:604-608.

-14 Graham GG, MacLean WC, Brown KH, et al: Protein requirements of infants and children: growth during recovery from malnutrition. Pediatrics 1996;97:499-505.

-15 Tomkins A, Garlick PJ, Schofield WN, Waterlow JC: The combined effect of infection and malnutrition on protein metabolism in children. Clin Sci 1983;65:313-324.

-16 Sokal EM, Baudoux MC, Collette E, et al: Branched chain amino acids improve body composition and nitrogen balance in a rat model of extra-hepatic biliary atresia. Pediatr Res 1996;40:66-71.

17 Chin SE, Shepherd RW, Thomas BJ, et al: The nature of malnutrition in children with endstage liver disease awaiting orthotopic liver transplantation. Am J Clin Nutr 1992;56: 164-168.

- 18 Koletzko B, Demmelmair H, Socha P: Nutritional support of infants and children: supply and metabolism of lipids. Baillieres Clin Gastroenterol 1998;12:671-696.

19 Beath S, Hooley, Willis K, et al: Long chain triacylglycerol malabsorption and pancreatic function in children with protein energy malnutrition complicating severe liver disease. Proc Nutr Soc 1993;52:252A.

20 Bach AC, Babayan V: Medium-chain triglycerides: an update. Am J Clin Nutr 1982;36: 950-962.

21 Kaufman SS, Murray ND, Wood RP, et al: Nutritional support for the infants with extrahepatic biliary atresia. J Pediatr 1987;10: 679-685.
-22 Rebouche CJ, Panagides DD, Nelson SE: Role of carnitine in utilization of dietary medium-chain triglycerides by term infants. Am J Clin Nutr 1990;52:820-824.

23 Kaufmann SS, Scrivner DJ, Murray ND, et al: Influence of portagen and pregestimil on essential fatty acid status in infantile liver disease. Pediatrics 1992;89:151-154

24 Bach A, Schirardin H, Bauer M, Weryha A: Ketogenic response to medium-chain triglyceride load in the rat. J Nutr 1977;107: 1863-1870.

25 Beath SV, Johnson S, Willis KD, et al: Growth and luminal fat solubilisation in cholestatic infants on medium chain triglyceride (MCT) J Pediatr Gastroenterol Nutr 1996;22:443.

26 Koletzko B, Agostoni C, Carlson SE, et al: Long chain polyunsaturated fatty acids (LC-PUFA) and perinatal development. Acta Paediatr 2001;90:460-464.

27 Koletzko B: Fats for brains. Eur J Clin Nutr 1992;46:S51-S62.

28 Demmelmair H, von Schenck U, Behrendt E, et al: Estimation of arachidonic acid synthesis in full term neonates using natural variation of C13 content. J Pediatr Gastroenterol Nutr 1995;21:31-36

29 Simmer K: Longchain polyunsaturated fatty acid supplementation in infants born at term (Cochrane Review); in The Cochrane Library, Issue 3. Cichester, Wiley, 2004.

30 Uauy R, Hoffman DR, Mena P, et al: Term infant studies of DHA and ARA supplementation on neurodevelopment: results of randomized controlled trials. J Pediatr 2003; 143:S17-S25.

-31 Birch EE, Hoffman DR, Castañeda YS, et al: A randomized controlled trial of long-chain polyunsaturated fatty acid supplementation of formula in term infants after weaning at 6 wk of age. Am J Clin Nutr 2002;75:570-580.

-32 Hoffman DR, Birch EE, Castañeda YS, et al: Visual function in breast-fed term infants weaned to formula with or without longchain polyunsaturates at 4 to 6 months: a randomized clinical trial. J Pediatr 2003;142: 669-677.

33 Field CJ, Van Aerde JE, Robinson LE, Clandinin MT: Effect of providing a formula supplemented with long-chain polyunsaturated fatty acids on immunity in full-term neonates. Br J Nutr 2008;99:91-99.

34 Levy J, DeFelice A, Lepage G: Essential fatty acid deficiency mimicking porphyria cutanea tarda in a patient with chronic cholestasis. J Pediatr Gastroenterol Nutr 1990;10: 242-245.

>35 Socha P, Koletzko B, Świątkowska E, et al: Essential fatty acid metabolism in infants with cholestasis. Acta Pediatr 1998;87:278283.

-36 Socha P, Koletzko B, Pawlowska P, Socha J: Essential fatty acid status in children with cholestasis, in relation to serum bilirubin concentration. J Pediatr 1997;131:700-706.
37 Socha P, Koletzko B, Pawlowska J, et al: Treatment of cholestatic children with water-soluble vitamin E (alpha-tocopheryl polyethylene glycol succinate): effects on serum vitamin E, lipid peroxides, and polyunsaturated fatty acids. J Pediatr Gastroenterol Nutr 1997;24:189-193.

-38 Socha P, Koletzko B, Świątkowska E, et al: Essential fatty acid metabolism in infants with cholestasis. Acta Pediatr 1998;87:278283.

39 Lapillonne A, Hakme C, Mamoux V, et al: Effects of liver transplantation on long-chain polyunsaturated fatty acid status in infants with biliary atresia. J Pediatr Gastroenterol Nutr 2000;30:528-532.

40 Hochman J, Balistreri WF: Neonatal cholestasis: differential diagnosis, evaluation and management; in Balistreri WF, Ohi R, Todani T, Tsuchida Y (eds): Hepatobiliary, Pancreatic and Splenic Disease in Children. Amsterdam, Elsevier, 1997.

41 Amedée-Manesme O, Furr HC, Alvarez F, et al: Biochemical indicators of vitamin A depletion in children with cholestasis. Hepatology 1985;5:1143-1148.

-42 Gumpricht E, Dahl R, Devereaux MW, Sokol RJ: $\beta$-Carotene prevents bile acid-induced cytotoxicity in rat hepatocyte: evidence for an antioxidant and anti-apoptotic role of $\beta$ carotene in vitro. Pediatr Res 2004;55:814821.

43 Leon AM, Ahmed S, Aleynik SI, et al: Carotenoids and tocopherols in various hepatobiliary conditions. J Hepatol 1995;23:550556.

44 Argao EA, Specker BL, Heubi JE: Bone mineral content in infants and children with chronic cholestatic liver disease. Pediatrics 1993;91:1151-1154.

45 Argao EA, Balistreri WF, Hollis BW, et al: Effect of orthotopic liver transplantation on bone mineral content and serum vitamin $\mathrm{D}$ metabolites in infants and children with chronic cholestasis. Hepatology 1994;20: 598-603.

46 Anderson CM, Townley RR, Freeman JP: Unusual causes of steatorrhea in infancy and childhood. Med J Aust 1961;11:617-621.

47 Nasr SZ, O’Leary MH, Hillermeier C: Correction of vitamin $\mathrm{E}$ deficiency with fat-soluble versus water-miscible preparations of vitamin $\mathrm{E}$ in patients with cystic fibrosis. J Pediatr 1993;122:810-812.

48 Sokol RJ, Kayden HJ, Bettis DB, et al: Isolated vitamin $\mathrm{E}$ deficiency in the absence of fat malabsorption - familial and sporadic cases: characterization and investigations of causes. J Lab Clin Med 1988;11:548-559.

49 Sokol RJ, Butler-Simon N, Conner C, et al: Multicenter trial of d-M-tocopheryl polyethylene glycol 1000 succinate for treatment of vitamin E deficiency in children with chronic cholestasis. Gastroenterology 1993;104: 1727-1735 
50 Sokol RJ, Heubi JE, Butler-Simon N, et al: Treatment of vitamin E deficiency during chronic childhood cholestasis with oral d-Ttocopheryl polyethylene glycol-1000-succinate. Gastroenterology 1987;93:975-985.

-51 Sokol RJ, Guggenheim MA, Iannaccone ST, et al: Improved neurologic function following long-term correction of vitamin E deficiency in children with chronic cholestasis. N Engl J Med 1985;313:1580-1586.

-52 Argao EA, Heubi JE: Fat-soluble vitamin deficiency in infants and children. Curr Opin Pediatr 1993;5:562-566.
53 van Hasselt PM, de Koning TJ, Kvist N, et al: The Netherlands Study Group for Biliary Atresia Registry. Prevention of vitamin K deficiency bleeding in breastfed infants: lessons from the Dutch and Danish biliary atresia registries. Pediatrics 2008;121:e857e863.

54 Amedée-Manesme O, Lambert WE, Alagille D, De Leenheer AP: Pharmacokinetics and safety of a new solution of vitamin $\mathrm{K}_{1}$ in chil dren with cholestasis. J Pediatr Gastroenterol Nutr 1992;14:160-165.

55 Bonkovsky HL, Banner BF, Lambrecht RW, Rubin RB: Iron in liver diseases other than haemochromatosis. Semin Liver Dis 1996; 16:65-82.

56 Wierzbicka A, Pawłowska J, Socha P, et al: Lipid, carbohydrate metabolism, and antioxidant status in children after liver transplantation. Transplant Proc 2007;39:15231525 .
57 Plauth M, Merli M, Weimann A, et al: ESPEN guidelines for nutrition in liver disease and transplantation. Clin Nutr 1997;16:4345.

58 Socha P, Koletzko B, Demmelmair H, et al: Short-term effects of parenteral nutrition of cholestatic infants with lipid emulsions based on medium-chain and long-chain triacylglycerols. Nutrition 2007;23:121-126.

59 Guimber D, Michaud L, Ategbo S, et al: Experience of parenteral nutrition for nutritional rescue in children with severe liver disease following failure of enteral nutrition. Pediatr Transplant 1999;3:139-145. 